\title{
Die Verantwortung der Wirtschaftsinformatik für unseren Planeten
}

\author{
DOI 10.1007/s11576-009-0180-z
}

\section{Die Autoren}

Prof. Dr. Hans Ulrich Buhl

Universität Augsburg

FIM Kernkompetenzzentrum

Finanz- \& Informationsmanagement

Universitätsstraße 16

86135 Augsburg

Deutschland

hans-ulrich.buhl@wiwi.

uni-augsburg.de

\section{Martin Jetter}

Vorsitzender der Geschäftsführung

IBM Deutschland GmbH

Pascalstraße 100

70569 Stuttgart

Deutschland

mjetter@de.ibm.com

This article is also available in English via http://www.springerlink.com and http://www.bise-journal.org:Buhl HU, Jetter M (2009) BISE's Responsibility for our Planet. Bus Inf Syst Eng. doi: 10.1007/s12599-009-0058-z.
Nach der Krise der New Economy durchleben wir mit der Finanzmarktkrise die zweite, noch wesentlich heftigere, Krise dieses noch jungen Jahrtausends. Bei der Untersuchung gemeinsamer Muster wird deutlich, dass wir - auf Individual-, Unternehmens- und auch staatlicher Ebene - systematisch Chancen überschätzt und damit einhergehend Risiken unterschätzt haben.

Zu Zeiten des New Economy Hypes wurde die Geschwindigkeit überschätzt, mit der das Internet Leben und Wirtschaft verändert. Dies hatte zur Folge, dass jedem Start-up mit Hightech-Bezug - ohne Beachtung möglicher Risiken - eine so goldene Zukunft prognostiziert und spätestens beim IPO so viel Liquidität zugeführt wurde, dass diesem viele weitere ohne klare Geschäftsaussichten folgten. Das Resultat dieser - gerade auch kapitalmarktseitigen - Übertreibung kennen wir: Zahlreiche Shakeouts, massive Kursrückgänge an den Weltbörsen insbesondere im Technologiebereich, die auf die Breite der Märkte ausstrahlten und in der Folge zu einem starken Anstieg der Arbeitslosigkeit führten.

Auch die Finanzmarktkrise, unter deren Folgen die Weltwirtschaft heute leidet, ist gekennzeichnet von einem nicht nachhaltigen, weltweiten Missmanagement von Chancen und Risiken. Diesmal in der Hauptrolle: Der Finanzdienstleistungssektor, der dank vermeintlicher Diversifikation sich und seinen Kunden mittels strukturierter Finanztitel wie ABS, MBS, CDOs, CDO2s, ABCPs, CDS Traumrenditen konstruierte, bis die nicht erkannten oder zumindest stark vernachlässigten Risiken dieser komplexen Instrumente zutage traten und ein massiver wirtschaftlicher Abwärtstrend einsetzte, der zum stärksten Welthandelsrückgang und Wirtschaftseinbruch zumindest seit dem zweiten Weltkrieg führte.

Auch wenn es auf den ersten Blick etwas verfrüht scheint, schon jetzt über die nächste Krise zu diskutieren - man braucht kein Hellseher zu sein: Sie kommt bestimmt! Und nach unserem Dafürhalten könnte diese Krise nicht nur einzelne Wirtschaftsbereiche treffen, sondern die Wirtschaft als Ganzes; dann nämlich, wenn sich Ressourcenpreissteigerungen zur nächsten Krise aufschaukeln: Je schneller die Energie- und Rohstoffpreise jetzt sinken, desto früher wird die nächste Krise eintreten, denn: Wenn als Folge der zu niedrigen Preise gepaart mit den Finanzierungsproblemen infolge der Finanzmarktkrise Investitionen in Ressourcenabbau, -verarbeitung, -einsparung und -substitution unterbleiben, steigt spätestens mit dem Wiederanstieg des Rohstoffbedarfs das Risiko massiver Preiserhöhungen und Anpassungsschocks, welche das zarte Pflänzchen der wiedererstarkenden Wirtschaft erneut in die Knie zwingen werden - sofern wir bis dahin nichts aus den letzen Krisen gelernt haben.

Stellen wir uns also die Frage, was auf individueller und auf Unternehmens-Ebene getan werden kann, dass uns die nächste Krise nicht genauso kalt erwischt wie die aktuelle. Was können insb. Unternehmen schon jetzt tun, um nicht unter der schon heute absehbaren künftigen Ressourcenpreiskrise zu leiden oder gar in ihr unterzugehen? Welche „to do"s lassen sich auf staatlicher Ebene identifizieren, um zukünftige Krisen proaktiv zu vermeiden, anstatt - wie aktuell zu beobachten - kapitalintensiv Feuerwehr spielen zu müssen, wenn das Wirtschaftsgebäude wieder lichterloh brennt? Und mit welchem Löschwasser - um im Bild zu bleiben - sollte dies angesichts der fünfstelligen Milliardenbeträge zur Bekämpfung der aktuellen Krise auch möglich sein? Und schließlich zu unserem Beitrag: Wie kann die Wirtschaftsinformatik all diese Akteure dabei unterstützen, sich gegen Folgekrisen wie die Ressourcenpreiskrise proaktiv zu immunisieren? 
Um im Folgenden Ursachen und Auswirkungen der sich anbahnenden Ressourcenpreiskrise genauer analysieren zu können, sind zunächst zwei Effekte zu unterscheiden, welche die Rohstoffpreisentwicklung bestimmen: Außer Frage steht, dass der Preis jedes endlichen Rohstoffs - abhängig von spezifischer Verfügbarkeit und spezifischem Verbrauch - langfristig exponentiell steigt. Geschuldet ist dies - neben einer immer kostspieliger werdenden Exploration - der Tatsache, dass sich ein abnehmendes Angebot in steigenden Preisen niederschlägt - und auch niederschlagen muss, um die Nachfrage kontinuierlich zu reduzieren. Unabhängig davon, wo die Reichweite eines Rohstoffs genau liegt (bei einigen Gewürzmetallen wie Indium oder Platin liegt die Schätzung aktuell bei 5 bis 15 Jahren), der langfristig exponentielle Preisanstieg ist jedem endlichen Rohstoff gemein. Überlagert wird dieser Preisanstieg von kurzfristigen Schwankungen, die u. a. konjunkturelle Ursachen haben oder sich auf Spekulationen zurückführen lassen. Bereits heute macht sich diese Volatilität an den Rohstoffmärkten - insbesondere bei bereits heute sehr knappen Rohstoffen - deutlich bemerkbar.

Selbst wenn die Prognose des Living Planet Report 2008 übertrieben scheinen mag, nach der die Menschheit - sofern deren Ansprüche an den Planeten weiter mit der gleichen Geschwindigkeit zunehmen - in den 2030er Jahren den Gegenwert von zwei Planeten brauchen wird, um ihren Lebensstil aufrechtzuerhalten, werden die Auswirkungen der auch von wissenschaftlicher Seite proklamierten stofflichen Grenzen des Wachstums (Christen) deutlich zunehmen. Schon heute ist gesundes, sicheres und sozial vertretbar angenehmes Leben nur für eine Minderheit der Erdbevölkerung möglich. So haben nur vier von fünf Menschen Zugang zu sauberem Trinkwasser. Das Bevölkerungswachstum wird diese Situation - mithin auch die damit zusammenhängenden Ressourcenengpässe - weiter und weiter verschärfen. Die Deutsche Stiftung Weltbevölkerung zählt pro Sekunde 2,6 Menschen hinzu. Derzeit wächst die Weltbevölkerung um über 80 Millionen Menschen pro Jahr - etwa so viele wie heute in Deutschland leben. Bis 2045 rechnen die Vereinten Nationen mit einem Zuwachs auf über 9 Milliarden Menschen. Der Wettlauf um Ressourcenzugang und Ressourceneffizienz wird sich erheblich verschärfen. Auf Dauer wird es sich die Menschheit also nicht leisten können, so kurzsichtig und ineffizient mit den zur Verfügung stehenden Ressourcen umzugehen, wie dies heute der Fall ist.

- Beispiel Energieversorgung: Nach Schätzungen der EU-Kommission sind in der Europäischen Union in den nächsten zwanzig Jahren Investitionen in einer Größenordnung von 1.000 Milliarden Euro erforderlich, um die alternde Infrastruktur zu ersetzen und den prognostizierten Energienachfrageanstieg zu decken. Aktuelle Investitionen in Kraftwerke, Übertragungs- und Verteilernetze sowie in neue marktfähige, effizientere und umweltverträgliche Technologien bleiben weit hinter diesem Bedarf zurück.

- Beispiel Wasser:Trinkwassernetze sind in Bau und Erhalt etwa dreimal so teuer wie Elektrizitätsnetze. Der größte Teil der Infrastruktur liegt im Untergrund - außer Sichtweite. Verluste durch Lecks werden auf 15 bis 20 Prozent geschätzt, in Entwicklungsländern deutlich darüber. Wir gehen mit einer unserer kostbarsten Ressourcen erstaunlich unbesorgt um.

- Beispiel Mobilität: Staus auf den Straßen der EU-Staaten kosteten 2007 rund 135 Milliarden Euro oder gut ein Prozent des gemeinsamen BIP.

Deswegen muss sich jeder rationale Rohstoffkonsument - sei es nun auf Individual-, Unternehmens- oder staatlicher Ebene - der absehbaren Auswirkungen der Ressourcenpreisschwankungen und -steigerungen auf seinen Verantwortungsbereich bewusst werden, um Strategien zu einer Reduktion der hieraus resultierenden Risiken entwickeln zu können. Wer es verpasst, sich bereits heute geeignet gegen die Folgen einer Ressourcenpreiskrise zu immunisieren, für den können diese leicht zum existentiellen Risiko werden.

Unserer Überzeugung nach kommt der Steuerungseffizienz der IT eine entscheidende Rolle zu, was sich auch in der Agenda „A Smarter Planet“ widerspiegelt, mit der IBM ihre Vision einer intelligent vernetzten Welt zusammenfasst. Durch den Einsatz intelligenter Systeme und Prozesse hilft die IT bspw. auf Unternehmensebene, relevante Informationen zur Verfügung zu stellen, die zu einer besseren Einschätzung der kurz- und langfristigen Entwicklung erfolgskritischer Ressourcenpreise führen und damit einhergehende Risiken sichtbar machen. Ziel der Wirtschaftsinforma- 
tik muss es sein, die Unternehmens-IT im Sinne eines ressourcenlenkenden Nervensystems zur „intelligenten Steuerung“ als Plattform einzusetzen und so - durch Erkennen von Knappheits- und damit Preisentwicklungsrisiken - zu einem Ertrags-/Risiko-effizienten Ressourcenmanagement zu gelangen, in dem das Zusammenspiel komplexer dezentraler Entscheidungen weit besser als heute koordiniert und damit nachhaltiger Unternehmenswert geschaffen wird.

Ausgangspunkt dieser Überlegungen sind die heute erreichte Durchdringung aller Lebens- und Arbeitsbereiche mit Technologie und die massive Vernetzung der dabei eingesetzten und zu immer geringeren Kosten verfügbaren Komponenten (Sensoren zum Messen, Aktuatoren zum Steuern, Embedded Systems) und Endgeräte (Computer, (Mobil-)Telefone, usw.). Physische Infrastruktur wird mit Intelligenz ausgestattet: Wir bauen „sehende, hörende, fühlende“ Straßen und Brücken, Pipelines und Leitungen, Gebäude und Fabriken. Jede Interaktion zwischen Mensch und Technologie sowie zwischen Komponenten oder Geräten birgt das Potenzial, einen Vorgang oder Ablauf effizienter, ressourcenschonender, sicherer, nützlicher, praktischer zu gestalten. Kurz: die Ubiquität der IT und die Konvergenz digitaler und physischer Systeme machen den Planeten smarter - vorausgesetzt sie werden mit der nötigen Intelligenz und Konsequenz eingesetzt.

Zwei Stellhebel sind näher zu betrachten: Erstens die Transparenz und bessere Steuerung des Energie- und Materialeinsatzes in der IT und zweitens die Einsparungen durch moderne IT-Anwendungen, die Geschäftsprozesse erneuern und energieeffizienter gestalten. Transparenz und Steuerung setzen voraus, dass die entsprechenden Verbräuche erfasst werden und nicht in Gemeinkosten oder umfassenden Liegenschaftsbudgets aufgehen. Welcher Rechenzentrumsleiter kennt heute seine Energiekosten? Welche Veranlassung hat er, energieeffizientere Layouts seiner Anlagen, einen anderen Energiemix oder geschicktere Verbrauchssteuerung anzustreben, wenn die entscheidenden KPIs nicht zur Verfügung stehen? Abhilfe schafft die Zertifizierung von Rechenzentren hinsichtlich ihrer Energieeffizienz. Der Kriterienkatalog umfasst umfangreiche Anforderungen an Klimatisierung, Stromversorgung, CO2-Emission und das Management des Rechenzentrums. Ziel ist es, die Verbindung von ökologischer und ökonomischer Effizienz nachvollziehbar und vergleichbar zu dokumentieren.

Auch wenn die Wirtschaftsinformatik nur eine Disziplin unter vielen ist, die ihren Beitrag zu nachhaltigem Wirtschaften zu leisten hat, wollten wir anhand dreier Beispiele auf Individual-, Unternehmens- und staatlicher Ebene schlaglichtartig verdeutlichen, wie dank IT ressourceneffizient und nachhaltig gewirtschaftet werden kann:

IBM befragte für ihre Verbraucherstudie mit dem Titel „Lighting the Way: Understanding the Smart Energy Consumer" 5.000 Stromkunden in zwölf Ländern - darunter Deutschland, Frankreich, Großbritannien, Japan, Australien, Kanada und die USA. Die erhobenen Daten verdeutlichen, dass mehr als 90 Prozent der Befragten sich nicht nur intelligente Stromzähler, sondern auch Instrumente zur besseren Steuerung ihrer Energienutzung wünschen. Zudem sind die Stromkosten und die Sorge um die Umwelt große Treiber für Veränderungen auf Kundenseite: Vier von fünf Befragten würden stromintensive Arbeiten im Haushalt auf andere Tageszeiten verlegen, wenn sie dabei günstigere Tarife in Anspruch nehmen könnten. Knapp 70 Prozent der Stromverbraucher erklären sich außerdem bereit, neue Programme und Services auszuprobieren, um ihre persönliche Ökobilanz zu verbessern.

Auch für die Energieversorger ist intelligente Steuerung des Energieverbrauchs ein großer Schritt nach vorn. Das Beispiel der Mittelmeerinsel Malta zeigt das deutlich. Ihre Unternehmen für die nationale Elektrizitäts- und Wasserversorgung - Enemalta Corporation und Water Services Corporation - implementieren während der nächsten fünf Jahre landesweit ein „Smart Grid“. Die Elektrizitäts- und Wassersysteme der Insel sind untrennbar verzahnt. Hinsichtlich der gesamten Stromproduktion und mehr als der Hälfte der Wasserversorgung, die der Filterung durch einen energieaufwendigen Entsalzungsprozess bedarf, ist Malta vollständig auf importiertes Erdöl angewiesen. Wenn Maltas Treibhausgasemissionen gesenkt und die Verfügbarkeit nachhaltiger Energie für die Zukunft gesichert werden sollen, ist eine intelligentere Energiewirtschaft unabdingbar. 250.000 analoge Stromzähler werden durch intelligente Messgeräte ersetzt, die den Elektrizitätsverbrauch in Echtzeit überwa- 
chen, undichte Stellen und Stromverluste identifizieren, variable Gebührensätze einstellen und besonders sparsame Kunden belohnen. Die Wasserzähler werden in ein System integriert, das Zählerstände und Rückstände überwachen und verwalten kann. Tausende von in die Infrastruktur integrierten digitalen Sensoren liefern Daten, die erfasst und analysiert werden. So kann die Regierung Maltas fundierte Entscheidungen hinsichtlich der Nutzung treffen und mit dem Ersatz des emissionsintensiven Erdöls durch nachhaltige Energiequellen beginnen.

Ein Beispiel für durch intelligente IT-Systeme verursachte ökonomische und ökologische Sekundäreffekte auf staatlicher Ebene ist die moderne Verkehrssteuerung. Mautsysteme werden vom Verbraucher meist als Bestrafungssystem empfunden und genießen folglich eher geringe Akzeptanz. Dass es auch anders geht, beweist die Stadt Stockholm, die nach erfolgreicher Pilotierung ihres neuen Verkehrsleitsystems die Bevölkerung vor dem weiteren Roll-out um ihre Meinung gebeten hat. Die Mehrheit sprach sich eindeutig dafür aus. Als ehemalige, aufgrund ihrer besonderen geografischen Situation von vielen Brücken, Umfahrungen und Engstellen geplagte und notorische Staustadt hat Stockholm heute eines der modernsten Verkehrsleitsysteme Europas. Bürger und Verwaltung erfreuen sich eines rund 20 Prozent geringeren Verkehrsaufkommens, einer Emissionsreduzierung von 12 Prozent und eines Nutzerzuwachses des öffentlichen Personennahverkehrs von rund 40.000 Menschen. Möglich wurde dies durch Vernetzung der Sensoren und Steuerungsinstrumente und durch automatische Nummernschilderfassung bei Einfahrt in die Innenstadt. Letztere erlaubt verkehrsvolumenabhängige Tarifierung und motiviert damit je nach "Stresslevel“ der Verkehrslage zum Umstieg auf den ÖPNV. Es sind solche und andere Referenzprojekte, die den Weg für einen intelligenteren Umgang mit Ressourcen weisen.

Und wie stehen die hier skizzierten, beispielhaften Maßnahmen im Zusammenhang mit der anfangs prognostizierten Ressourcenpreiskrise? Ganz einfach: Wem es - insbesondere mit der Steuerungseffizienz der IT - gelingt, Ressourcen einzusparen bzw. effizient einzusetzen, der ist weniger abhängig von den kurzfristigen Schwankungen und langfristig exponentiellen Steigerungen der Rohstoffpreise. Dies gilt bspw. für US-amerikanische Verbraucher, die statt eines bisherigen Kaufs eines SUV mit dem Luftwiderstand eines Kühlschranks, dem Rollwiderstand eines Treckers, aber 250 km/h Spitzengeschwindigkeit aufgrund eines großvolumigen Achtzylinder-Benzinmotors auf sparsamere bzw. Hybrid-Technologien umsatteln. Es gilt ebenso für Unternehmen und Staaten, die sich bereits heute gegen die kurz- und langfristigen Risiken der sich anbahnenden Ressourcenpreiskrise zu immunisieren beginnen. Und schlussendlich wird die Wirtschaft - global betrachtet - durch jedes Wirtschaftssubjekt stabilisiert, welches sich heute wirksam und nachhaltig gegen Ressourcenpreisrisiken immunisiert. Die daraus resultierende Gesamtrisikoreduktion wird entscheidend für die Stabilisierung der Weltwirtschaft und somit zur Verhinderung neuer Krisen sein. Deswegen muss es unser aller Ziel sein, dass eine kritische Masse an Einzelpersonen, Unternehmen und Volkswirtschaften einen intelligenten Beitrag zur effizienten und sparsameren Nutzung endlicher Rohstoffe leistet. Denn: Eine Weltwirtschaft, die hohen Risiken ausgesetzt ist - z. B. einer hohen Volatilität von Ressourcenpreisen, Nahrungsmitteln, Energiepreisen, etc. - macht hauptsächlich Spekulanten reich; die Verlierer dabei sind stets die Schwächsten. Jeder, der sich heute dieser Verantwortung nicht stellt, wird sich spätestens in der nächsten Krise den Folgen seines nicht nachhaltigen Verhaltens stellen müssen.

Prof. Dr. Hans Ulrich Buhl Martin Jetter

\section{Literatur}

Christen M (2005) Die stofflichen Grenzen des Wachstums. http://www.physik.uniaugsburg.de/chemie/ pressespiegel/pdf/die_stofflichen_grenzen_des_wachstums.pdf. Abruf am2009-05-04

Leape JP (2008) Living Planet Report 2008. http://www.wwf.de/fileadmin/fm-wwf/pdf_neu/Living_ Planet_Report_2008_WWF.PDF. Abruf am 2009-05-04 


\section{Business oder Technologie?}

\section{Wieso oder?}

\section{Oder kennen Sie ein Business,}

das ohne Technologie auskommt?

McKinsey sucht Berater(innen) für das Business Technology Office.

Der effektive Einsatz von Technologie löst unternehmerische Probleme und steigert somit den substanziellen Wert eines Unternehmens nachhaltig. Genau das ist das Ziel unserer Beratung. Wir helfen unseren Klienten, Investitionen in Technologie erfolgreich zu managen und mit den strategischen Prioritäten des Geschäfts in Einklang zu bringen. Wenn Sie diese vielfältige Herausforderung annehmen, werden Sie auf über 500 Kollegen an rund 50 Standorten in mehr als 25 Ländern treffen. Und werden damit Teil des globalen Business Technology Office - eines der größten und am schnellsten wachsenden Büros von McKinsey \& Company. Alle weiteren Informationen finden Sie auf unserem Karriereportal. 